\title{
La especialización hemisférica y la regulación de la conducta motora desde la perspectiva de la neurociencia cognitiva
}

\author{
Sergio Machado, 1,2,3,7 Oscar Arias-Carrión, ${ }^{4}$ Alejandra Verónica Orellana Castillo, ${ }^{5}$ Eduardo Lattari, ${ }^{6}$ \\ Adriana Cardoso Silva, ${ }^{1}$ Antonio Egídio Nardi ${ }^{1}$
}

Actualización por temas

\section{SUMMARY}

\section{Introduction}

Our understanding of functional brain organization is due to advances in neuroimaging technologies and intensive clinical research. Recently, cognitive science (cognitive neuroscience), combined with advances in technology, have changed our understanding of brainbehavior relationship. This symbiotic relationship has allowed a better characterization of the lesion site in patients with brain disorders and patterns of activation in healthy subjects.

\section{Objective}

In this article we discuss the contribution of the left hemisphere and right hemisphere involvement in the regulation of motor behavior; this will allow us to better understand the lateralization of motor functions.

\section{Development}

The results support the view of a left hemisphere dominance for language and motor control, and a right hemisphere dominance for spatial functions and attention. Specialized areas are probably predetermined and certain functions are lateralized to one or other hemisphere due to the efficient organization and information processing in the brain.

\section{Conclusion}

In the studies reviewed, specific functions for each hemisphere were observed, suggesting the existence of a complex organization that recruits several areas of the Nervous System for proper task performance.

Key words: Cognitive neuroscience, functional integration, functional specialization, motor behavior, sensorimotor integration.

\section{RESUMEN}

\section{Introducción}

Nuestra comprensión de la organización funcional del cerebro se debe a los avances en las técnicas de neuroimagen y a una intensa investigación clínica. Recientemente, la ciencia cognitiva (neurociencia cognitiva) en combinación con los avances tecnológicos han cambiado nuestra comprensión sobre la relación cerebro-conducta. Esta relación simbiótica ha permitido una mejor caracterización del sitio de la lesión en pacientes con trastornos cerebrales y de los patrones de activación en sujetos sanos.

\section{Objetivo}

En el presente artículo se discute la contribución del hemisferio izquierdo y la participación del hemisferio derecho en la regulación de la conducta motora; esto nos permitirá comprender mejor la lateralización de las funciones motoras.

\section{Desarrollo}

Los resultados apoyan la visión de un predominio del hemisferio izquierdo para el lenguaje y el control motor, y un predominio del hemisferio derecho para las funciones espaciales y la atención. Las áreas especializadas son probablemente predeterminadas y ciertas funciones están lateralizadas a uno u otro hemisferio, esto debido a la eficiente organización y procesamiento de la información en el cerebro.

\section{Conclusión}

En los estudios revisados, se observaron funciones específicas para cada hemisferio, lo que sugiere la existencia de una compleja organización que recluta a varias áreas del Sistema Nervioso para el adecuado desempeño de una tarea.

Palabras clave: Conducta motora, especialización funcional, integración funcional, integración sensitivomotora, neurociencia cognitiva.

Laboratory of Panic and Respiration, Institute of Psychiatry, Federal University of Rio de Janeiro (UFRJ), Rio de Janeiro, RJ, Brazil; National Institute of Translational Medicine (INCT-TM), Brazil.

2 Quiropraxia Program of Faculty of Medical Sciences, Central University (UCEN), Santiago, Chile.

Institute of Philosophy, Federal University of Uberlândia, Minas Gerais, Brazil.

Movement Disorders and Transcranial Magnetic Stimulation Unit, Hospital General Dr. Manuel Gea González. México.

Fundación Duoc. Pontifícia Universidad Católica de Chile, Santiago, Chile.

Programa de Pós Graduação em Ciências do Esporte e do Exercício da Universidade Gama Filho- UGF, Rio de Janeiro, RJ, Brasil; Laboratório de Neurociência do Exercício- UGF, Rio de Janeiro, RJ, Brasil.

Laboratório de Neurociência da atividade Física, Programa de Pós-Graduação em Ciências da Atividade Física (PPGCAF), Universidade Salgado de Oliveira, Niterói, Brasil.

Correspondencia: Sergio Machado, PhD. Rua Bolivar, 150/apto. 702. Copacabana-Rio de Janeiro, RJ - Brazil. Cep 22061-020. E-mail: secm80@yahoo.com.br

Recibido: 31 de enero de 2013. Aceptado: 30 de agosto de 2013. 


\section{INTRODUCCIÓN}

El progreso en la comprensión de la organización funcional del cerebro se debe a los avances en las técnicas de neuroimagen y a una intensa investigación clínica. Recientemente la ciencia cognitiva, específicamente la neurociencia cognitiva, combinada con numerosos avances, ha logrado un gran impacto en nuestra comprensión sobre las relaciones cerebro-conducta. Esta relación simbiótica entre la neuropsicología y la neurociencia continúa y se ha logrado gracias al desarrollo de los métodos de neuroimagen. Esto ha permitido una mejor caracterización del sitio de la lesión en pacientes con trastornos cerebrales y de los patrones de activación en sujetos sanos. Esta simbiosis es especialmente atractiva cuando se combina con los paradigmas cognitivos con los más avanzados métodos de neuroimagen con el fin de abordar las cuestiones relacionadas con la cognición y su relación con aspectos neuroanatómicos. ${ }^{1}$

La complejidad del cerebro hace que este órgano se vea como un gran sistema abierto que integra muchos sistemas diferentes y complejos. Las modernas técnicas de neuroimagen nos permiten observar en vivo el funcionamiento del cerebro en tiempo real durante la ejecución de tareas cognitivas o de conducta. Esto lleva a una visión más actual e integrada del cerebro, donde múltiples áreas sean simples o complejas se activan al mismo tiempo. ${ }^{2}$ Basándose en esta visión de vanguardia, los investigadores se han esforzado para apoyar los principios que rigen la participación cortical desde la perspectiva de la funcionalidad. ${ }^{3-6}$ Por lo tanto, se han propuesto dos principios fundamentales: la especialización funcional, que se refiere a la idea de que determinadas regiones del cerebro desempeñan funciones especializadas, y la integración funcional, lo que implica que las tareas específicas requieren amplias interacciones entre regiones especializadas del cerebro. ${ }^{7}$

Tradicionalmente la neurociencia cognitiva depende de la información de estudios en animales y de investigación en pacientes con lesiones cerebrales focales con el fin de determinar el papel específico de las áreas. El primer método de investigación tiene el inconveniente de generalizar los resultados debido a los diferentes tipos de especies utilizadas en los estudios y también al hecho de que muchas funciones cognitivas de orden superior son inherentes a cada ser humano. El segundo método de investigación tiene el problema de que la lesión no suele ser focal y lo que se está investigando es el resto de la función cerebral después de una reorganización a largo plazo con falta de contribución del área lesionada. ${ }^{1}$ Con respecto a los estudios de las lesiones corticales, la doctrina localizacionista contribuyó eficazmente en los aspectos de la diferenciación funcional de los hemisferios cerebrales. Desde los estudios de Paul Broca y de Carl Wernicke se observó que las áreas del lenguaje se alteran cuando hay una lesión específica en el hemisferio izquierdo. De acuerdo con esto, Hughlings Jackson dirigió la discusión por medio de de sus experimentos para un posible predominio del hemisferio derecho debido a su importancia en las funciones visuo-espaciales. ${ }^{8}$ Por el contrario, Liepmann señaló una notable asimetría inter-hemisférica en relación con las habilidades motoras, con el argumento de que el hemisferio izquierdo juega un papel preponderante en el control de movimiento, rectificando que este hemisferio contiene "fórmulas de movimiento" destinadas a ambos lados del cuerpo. ${ }^{9}$

Basándose en estas discusiones, en la actualidad la neurología ha concentrado su atención en las funciones especializadas del hemisferio izquierdo que son esenciales para el lenguaje y habilidades motoras. Los estudios demuestran que esta lateralización está bien establecida para los sujetos diestros ${ }^{10-12}$ y que esto podría ser programado durante el desarrollo ${ }^{13,14}$ después de la aparición de las especializaciones perceptivo-motoras esenciales en el período inicial del embarazo. ${ }^{15-17}$ Sin embargo, este tema aún ha generado debates. ${ }^{18,19}$ Una posible hipótesis es que las conexiones funcionales entre las áreas corticales de la mano y la lengua ${ }^{20,21}$ pudieron haber sido esenciales para la evolución del lenguaje de gestos con las manos en lugar de palabras vocales, ${ }^{22}$ un hecho que es apoyado por el uso de gestos que suelen acompañar a la expresión del discurso. ${ }^{23}$ En este contexto, algunos estudios han encontrado que el área de Broca se asocia con diferentes funciones motoras y no sólo con el lenguaje, sino también con la planificación, el reconocimiento y la imitación de ges$\operatorname{tos}^{24-26}$ y las operaciones sintácticas necesarias para la representación jerárquica de la conducta secuencial. ${ }^{27,28}$

El objetivo de este artículo es discutir las contribuciones del hemisferio izquierdo en la regulación de la conducta motora y la participación del hemisferio derecho en ese proceso para comprender mejor sus participaciones en la lateralización de las funciones motoras. Por lo tanto, se propone que la lateralización de las funciones motoras es un proceso versátil en el que la participación funcional de uno y otro no es fijo, sino flexible y guiada por varios factores fundamentales.

\section{CONTRIBUCIONES DE LOS HEMISFERIOS IZQUIERDO Y DERECHO EN LA REGULACIÓN DE LOS ASPECTOS COGNITIVOS DE LA CONDUCTA MOTORA}

Los expertos en neurología y neuropsicología típicamente enfocan su atención en los déficits motores contralesionales, pero los déficits ipsilesionales también están presentes y son considerados como asociados con las demandas cognitivas de los movimientos (por ejemplo, la planificación, el desarrollo y la selección de los programas motores). Estas deficiencias tienen implicaciones funcionales, especialmente en pacientes con secuelas de accidente cerebrovascular (ACV) que utilizan sus extremidades ipsilesionales para compensar la hemiplejía. Estudios anteriores han puesto de relieve la importancia del hemisferio izquierdo en comparación con el derecho para el control del movimiento ipsilesional, por ejemplo, por el 
miembro de un paciente con apraxia ${ }^{29}$ y en secuencia de movimientos. ${ }^{30,31}$ Algunas tareas son más controladas por el hemisferio derecho o bien requieren ambos hemisferios, ${ }^{32}$ pero el foco de la discusión es el papel del hemisferio izquierdo en los movimientos complejos, ya que es dominante en el control de muchas acciones, tales como el lenguaje.

La especialización hemisférica está estrechamente vinculada con la dominación hemisférica y las funciones cerebrales. El predominio del hemisferio izquierdo para las habilidades motoras se ha atribuido a las asimetrías anatómicas y funcionales de la corteza motora primaria (M1) y las vías descendentes $^{33,34}$ al igual que a las áreas motoras secundarias y de asociación..$^{35} \mathrm{El}$ mapa contralateral de la mano en los sujetos diestros proporciona una fuerte evidencia de una asimetría en el área $\mathrm{M} 1{ }^{36-39}$ que está probablemente relacionada con los cambios experimentados en el desarrollo temprano. Este hecho se observó en el desempeño de las tareas con la mano dominante y la no dominante en sujetos diestros, lo que indica una mayor dispersión espacial en las áreas motoras del hemisferio izquierdo que en las del derecho. ${ }^{40}$ Estudios con resonancia magnética funcional (RMf ${ }^{41}$ y estimulación magnética transcraneal (ETM) ${ }^{42}$ han mostrado los aspectos anteriormente mencionados.

La asimetría de las áreas motoras y de asociación refleja una aplicación secundaria de determinadas funciones de las regiones especializadas del cerebro. Esto se observó en estudios con pacientes que sufrieron lesiones en el hemisferio izquierdo. Los individuos mostraron una disminución en el desempeño de acciones hábiles con ambas manos. Las lesiones del hemisferio derecho producen déficits restringidos a la mano contralateral. ${ }^{43-45}$ Haaland et al. ${ }^{36}$ demostraron, en personas sanas, una mayor participación de las áreas pre-motoras y del hemisferio parietal izquierdo en comparación con el hemisferio derecho en áreas de alto comando que se relacionan con la complejidad de la tarea. De acuerdo con estos hallazgos, se han ofrecido varias hipótesis como base para este modelo funcional asimétrico del hemisferio izquierdo como la conducta de las acciones secuenciales, la organización y selección de movimientos, ${ }^{46,47}$ la coordinación bimanual, ${ }^{48,49}$ la percepción y la interpretación de acciones, ${ }^{50}$ y por otra parte, los movimientos en secuencia ${ }^{51}$ que refuerzan el papel del hemisferio izquierdo en el lenguaje hablado y escrito. ${ }^{52,53}$

Pero el verdadero papel del hemisferio derecho en la regulación de la conducta motora aún no está bien definido. La evidencia apunta a una representación reducida en el área M1 del hemisferio derecho en comparación con el izquierdo en las personas diestras, relacionado con una disminución de la destreza de la mano no dominante. ${ }^{54}$ En las áreas de asociación, las funciones especializadas relacionadas con la planificación no están muy desarrolladas, ${ }^{55}$ probablemente debido a la fuerte demanda de "pistas" para seleccionar una determinada representación externa que involucra el procesamiento motor de la nueva exploración de situaciones. ${ }^{56} \mathrm{El}$ tema del procesamiento de la exploración de nuevas situaciones es consistente con la teoría de que el hemisferio izquier- do actúa en aspectos del movimiento en circuito abierto (en base a programas motores bien establecidos), mientras que el hemisferio derecho es crucial para todos los aspectos del movimiento en circuito cerrado (dependiendo de la retroalimentación sensorial). ${ }^{38}$ Sin embargo, el estudio de Haaland et al. ${ }^{37}$ no proporciona ninguna evidencia de que esta dicotomía se relacione con la asimetría hemisférica. Por otra parte, se ha propuesto que el hemisferio izquierdo controla la trayectoria de la extremidad, mientras que el hemisferio derecho regula la posición y la postura de la extremidad utilizada en la tarea. ${ }^{57}$ Esta premisa es consistente con estudios realizados en pacientes que mostraron una conducta diferente en los casos de lesiones en los hemisferios izquierdo y derecho, al principio y al final del movimiento con el objetivo direccionado. ${ }^{58}$

Por lo tanto, hasta ahora lo más probable es que la especialización del hemisferio izquierdo se vea limitada por las especificaciones de la retroalimentación de tareas dinámicas, mientras que la especialización del hemisferio derecho incluye mecanismos sensoriales que controlan el posicionamiento final del miembro. ${ }^{59}$ Sin embargo, hay autores que mencionan al hemisferio derecho como encargado de funciones como la memoria espacial, el aprendizaje y la orientación, ${ }^{57}$ lo que sugiere que su predominio se debe al almacenamiento de características globales, mientras que la especialización del hemisferio izquierdo se produce por las características específicas de procesamiento. ${ }^{60}$ Por lo tanto, la especialización del hemisferio derecho en funciones espaciales puede deberse a la participación del control de la atención espacial en los campos de visión izquierdo y derecho ${ }^{61}$ o una función de supervisión que se hace evidente sobre todo en situaciones de conflicto ${ }^{62}$ entre la intención del movimiento, la propiocepción y la retroalimentación visual. ${ }^{63}$

\section{LA INTERACCIÓN ENTRE LOS FACTORES RELACIONADOS CON LA TAREA Y CON EL EJECUTOR}

Entender el funcionamiento del Sistema Nervioso Central (SNC) respecto a la regulación del movimiento es de gran importancia en varias áreas de investigación. Siguiendo esta línea vamos a discutir el que la participación de cada hemisferio cerebral en el control del movimiento depende principalmente de los aspectos relacionados con la tarea y el ejecutor. Por lo tanto, hay que señalar el tipo y la complejidad del movimiento, el nivel de habilidad del ejecutor, si el SNC ha sufrido algún daño o no y el foco de atención del ejecutor, ya que estos factores influyen en cómo los hemisferios se comportarán en la regulación del movimiento.

\section{Factores relacionados con la tarea}

El proceso relacionado con el tipo de movimiento tiene un papel importante en los mecanismos de regulación de la 
conducta motora. En particular, las representaciones secuenciales y sus características están asociadas con el hemisferio izquierdo, independientemente de la mano utilizada en la tarea. ${ }^{36}$ Así, el hemisferio izquierdo puede estar involucrado específicamente en la planificación de actos secuenciales que resultan en la selección de respuesta, la preparación y/o recuperación. ${ }^{36,64}$ Para alcanzar las metas específicas con un propósito, se propone que cada hemisferio contribuye de manera diferente en el control específico de la trayectoria y la posición final. ${ }^{59,65}$ Esta distinción se debe a la contribución del hemisferio izquierdo en la planificación de la dinámica de los miembros, mientras que el derecho es esencial para especificar la posición final de los movimientos de aprehensión a través de la regulación sensorial. La contribución de cada hemisferio también es modulada por la complejidad del movimiento. Mientras que uno simple, como doblar un dedo, es organizado por una red neuronal local, las acciones más complejas, tales como demandas relacionadas con una secuencia de movimientos de los dedos, requieren la participación distribuida de redes neuronales, a menudo de forma bilateral. ${ }^{36,64}$ En este sentido, el reclutamiento de ambos hemisferios parece ser afectado por el aumento de la atención o los parámetros de control ejecutivo, o por el uso de las operaciones que se especializan en cada hemisferio. Por lo tanto, se sabe que las vías entre los hemisferios tienen en cuenta el acoplamiento o desacoplamiento de las informaciones relevantes. ${ }^{66}$ En realidad, esta entrada de información se produce entre ambos hemisferios, como se ve en los estudios de transferencia de aprendizaje que investigan la transmisión de información cuando una tarea específica se realiza con una sola mano, dado que, en general, las ventajas de rendimiento se encuentran tanto en la mano entrenada como en la mano no entrenada. ${ }^{67}$

\section{Factores relacionados con el ejecutor}

El proceso relacionado con el ejecutor tiene gran importancia en la regulación de la conducta motora. Según estos principios, el experimento de Goldberg et al. ${ }^{68}$ propuso que el procesamiento del hemisferio derecho es impulsado por el ambiente externo, mientras que el procesamiento del hemisferio izquierdo se guía por las representaciones internas. ${ }^{37,69}$ Esto es consistente con las observaciones realizadas en los estudios donde los pacientes con lesiones en la corteza parietal derecha presentaban negligencia espacial. En ellos se ha observado un cambio severo de los movimientos de exploración hacia el lado derecho, que se atenuó cuando los movimientos fueron dirigidos hacia un objetivo. ${ }^{70}$ Se sugiere que ambos tipos de acción requieren una entrada de información diferente o procesos de apoyo con una aportación característica de ambos hemisferios. Estas diferencias funcionales entre hemisferios apoyan un cambio desde la derecha hacia la izquierda en la importancia hemisférica, tales como el desarrollo de habilidades. De hecho, este desarrollo se asocia a menudo con una transición parcial en la generación de control del movimiento de forma externa a interna. Este hecho se produce, por ejemplo, al aprender una tarea bimanual difícil, ${ }^{6}$ ocurriendo una disminución de la activación en el hemisferio derecho con el tiempo, mientras que la activación del hemisferio izquierdo se hace más inminente. Dado que la disminución de la activación en el hemisferio derecho se relaciona con una menor demanda de las características espaciales de seguimiento de movimiento, el aumento de participación y representación queda consolidada. . $^{670}$

El SNC presenta cambios operativos debido a ciertos patrones neuronales. Un ejemplo de esto sucede después de una lesión, en particular en las áreas corticales asociadas con el control bilateral de una determinada función como la corteza pre-motora que puede asumir más responsabilidades en el procesamiento motor, además de su papel crucial en la recuperación funcional. ${ }^{58,71}$ Esto implica que ambos hemisferios están dotados de las capacidades funcionales que pueden desempeñar bajo condiciones específicas y apoya la idea de que la participación de ciertas redes neuronales para la regulación de la conducta motora es flexible, aunque hay pruebas de que el déficit motor difiere según que las lesiones estén en el hemisferio izquierdo o en el derecho. ${ }^{72}$ Esta cuestión requiere una evaluación más detallada de lo que se refiere a un lado y la extensión de la lesión, así como las limitaciones de la tarea y la lateralidad.

Por último, la atención puede modular la participación de ambos hemisferios. ${ }^{73}$ En particular, los cambios en la atención espacial entre el nivel global y local de la representación dependen de los procesos relacionados con los hemisferios izquierdo y derecho, respectivamente. Además, estos cambios también dependen de las demandas relevantes y no al movimiento voluntario, ya que la atención dirigida al movimiento mejora la selección de las representaciones. Estos hallazgos demuestran que las demandas de las tareas y las características del ejecutor son factores que influyen en la regulación de la conducta motora y, por lo tanto, pueden promover las asimetrías hemisféricas y las interacciones inter-hemisféricas. Estos hechos demuestran que un equilibrio dinámico entre las restricciones existentes induce a ciertos modos de operación. ${ }^{6}$ Por ejemplo, se puede citar la orientación general particularmente relevante para las acciones nuevas e inexploradas más asociadas con el hemisferio derecho. Por otro lado, se pueden mencionar los tratamientos relacionados con las representaciones que se producen debido a una experiencia basada en una planificación de la acción, la mayoría relacionados con el hemisferio izquierdo. Estos hallazgos sustentan la contribución funcional de que ambos hemisferios son flexibles. Y es esta flexibilidad lo que produce una conducta motora adaptada y especializada. Por lo tanto, la observación de tareas motoras, por ejemplo los movimientos secuenciales de los dedos, es ampliamente utilizada en varios tipos de experimentos permitiendo obtener resultados en favor de los procesos por separado (principalmente del hemisferio izquierdo). ${ }^{1,6}$ 


\section{Integración sensitivomotora y procesamiento de la información}

La integración sensitivomotora puede definirse como un proceso mediante el cual los estímulos sensoriales se convierten en órdenes motoras, la información sensorial se integra por el SNC y es utilizada para ejecución de programas motores..$^{56}$ En este caso, el SNC procesa la información de múltiples canales sensoriales y se adapta al medioambiente que permite la ejecución de tareas específicas y de los movimientos con objetivos direccionados. ${ }^{74,75}$ Por lo tanto, la producción de un movimiento consiste en la integración entre los diferentes sentidos, especialmente la visión, la audición, la somestesia, etc. En las últimas décadas se han realizado varios experimentos con el objetivo de aclarar las áreas y procesos del cerebro implicados en la integración de la información sensorial y la ejecución del gesto motor. Werhahn et al. ${ }^{76}$ encontraron, por medio del uso de la EMT, que cuando se anestesiaba la extremidad superior contralateral de los sujetos, los músculos ipsilaterales sufrían un aumento en los potenciales motores evocados (PEM). Por otra parte, se observó que hubo una reducción en la excitabilidad de la corteza motora del hemisferio contralateral de la extremidad anestesiada frente a la excitabilidad de la corteza motora del hemisferio relacionado con el miembro no anestesiado.

De acuerdo con estos principios, si ciertas funciones son lateralizadas a ambos hemisferios, el procesamiento eficiente de información es esencial para la representación de dichas funciones. Muchas de estas interacciones se producen a través del cuerpo calloso teniendo en cuenta la transferencia de información pre-motora relacionada con la atención, la retroalimentación y los errores. ${ }^{77}$ Esta comunicación interhemisférica implica tanto una inhibición como una facilitación funcional. Aunque estas interacciones inhibitorias sean esenciales en la preparación de los movimientos unilaterales, éstas son responsables de neutralizar la producción de movimientos espejados, ${ }^{49,78}$ es decir, movimientos involuntarios de la mano contralateral, que lleva a cabo acciones de voluntariado. Desde el punto de vista de la lateralización de la conducta motora, hay evidencias de muchos circuitos inhibitorios entre la corteza motora del hemisferio izquierdo y derecho en sujetos diestros. ${ }^{79}$ Este tipo de distinción funcional podría contribuir a las diferencias hemisféricas en la regulación de la conducta motora, probablemente a partir de la infancia, durante ciertos procesos que asumen un comportamiento inhibitorio asimétrico. ${ }^{80}$

Facilitar la transferencia de información entre los hemisferios es esencial cuando su procesamiento sea necesario para un desempeño satisfactorio en la tarea. En el contexto de la regulación de la conducta motora, el cuerpo calloso y su mecanismo de intercambio de información ha sido visto con especial interés en la coordinación de las tareas bimanuales. ${ }^{81}$ Como se ha demostrado hasta el momento, el grado de comunicación interhemisférica está relacionado con la complejidad de la tarea ${ }^{3,6}$ y es necesario para el aprendizaje de nuevos patrones motores.,3,5 Así, las interacciones del cuerpo calloso proporcionan un enlace para la especificación de parámetros motores de alto nivel (tales como la velocidad con que actúa el miembro implicado en el movimiento) o la selección de la respuesta ${ }^{82,83} \mathrm{y}$, probablemente, constituyen una base fisiológica para el intercambio de información neural ${ }^{84}$ que se hace evidente cuando se participa en tareas bimanuales con diferentes patrones de movimiento. ${ }^{85}$ En resumen, esta conducta motora de adaptación depende de los procesos inhibitorios que pueden ayudarle a explorar los beneficios asociados con la transformación de la especialización hemisférica y facilitar procesos que permitan la integración de información en ambos hemisferios. Esto implica que la asignación de recursos de procesamiento para la conducta motora es un proceso dinámico por el cual la segregación y la integración de estas funciones se producen de una manera flexible.

Sin embargo, nuestra pregunta es: ¿cómo organiza el cerebro la información de diferentes regiones especializadas, ya que cada una puede procesar la información de diferentes maneras? Para comprender mejor esto es importante considerar cómo el procesamiento de información se coordina interhemisféricamente. Un posible mecanismo de las interacciones entre regiones de gran escala es la creación temporal de conexiones dinámicas que se basan en la sincronización de la actividad neural. ${ }^{86-88}$ En este sentido, la función de coherencia es vista como relevante por varios estudios electrofisiológicos como electroencefalografía, magnetoencefalografía y potenciales de campo local. ${ }^{89-91}$ En particular, la coherencia es un medio para captar la comunicación neuronal a través de diversos sectores de la corteza cerebral de codificación de información diferente que pueden estar relacionadas con diferentes bandas de frecuencia. A modo de ejemplo, la banda de frecuencias beta $(\sim 14-30 \mathrm{~Hz})$ se relaciona con la actividad sincronizada observada en las áreas sensitivomotoras. ${ }^{92}$ Así, la organización dinámica de la actividad neuronal en el dominio de la frecuencia puede proporcionar un medio de procesamiento de datos teniendo en cuenta las exigencias de la tarea tales como la estrecha relación entre los cambios en la coherencia corticocorticales y los resultados de la conducta, lo que apoya este punto de vista. ${ }^{93}$ Con base en estos principios, el estudio de Andrés et al. ${ }^{94}$ encontró que el aumento inicial de la coherencia puede ser un reflejo de los cambios en la comunicación interhemisférica que están específicamente relacionados con el aprendizaje bimanual y éstos pueden ser transmitidos a través del cuerpo calloso. Estos resultados pueden ayudar a explicar algunos datos neurofisiológicos de la observación clínica de los pacientes con lesiones del cuerpo calloso, que puede mostrar el resultado de déficit en la adquisición de nuevas tareas bimanuales, pero no necesariamente en la ejecución de las actividades bimanuales previamente aprendidas.

Esta organización dinámica también sugiere que la actividad de varias áreas del cerebro armonizadas representa un modo básico de comunicación con diferentes frecuencias que se basa en la formación de redes neuronales lo que traduce a 
la salida de la transformación en una conducta más eficiente. En cuanto a las pruebas presentadas en los temas anteriores, se sugiere que con el desarrollo de habilidades, las representaciones motoras, tales como las que se establecen en el hemisferio izquierdo, pueden apoyar con más efectividad el procesamiento de la información dentro de distintas regiones cerebrales. Un ejemplo es que durante la adquisición de una nueva tarea bimanual se ha demostrado que un perfil inicial de las asociaciones entre las áreas corticocorticales se ajusta gradualmente a medida que la rutina se convierte en un rendimiento estable y de conducta optimizada ${ }^{5,93,94}$ hasta que finalmente resulta en un patrón funcional que es sobre todo orquestado por el hemisferio izquierdo durante la ejecución de las tareas bimanuales aprendidas. ${ }^{3,5,6}$

\section{LA LATERALIZACIÓN DE LAS FUNCIONES MOTORAS: ¿ES REALMENTE UN PROCESO DINÁMICO?}

La participación funcional tanto del hemisferio izquierdo como del hemisferio derecho en la regulación de la conducta motora parece no ser fija, sino más bien un proceso dinámico. Dentro de este contexto, se aborda en esta sección una nueva perspectiva sobre la dinámica de este proceso y argumentando también que la lateralización de la función motora se caracteriza por diferentes canales de comunicación y dinámica intra e interhemisférica. De acuerdo con esto, los estudios de neuroimagen y los estudios clínicos con pacientes ayudan a identificar áreas y sistemas neuronales críticos para los procesos cognitivos en particular. ${ }^{95}$ Sin embargo, los estudios en pacientes con lesión cerebral están limitados por la incapacidad de controlar la ubicación de la lesión, el tamaño y la tendencia a centrarse en la importancia de un espacio único en lugar de un número de áreas que pueden ser críticas y pueden formar un circuito nervioso que controla una función compleja.

En este sentido, una cuestión importante e interesante es si dos o más áreas pueden controlar diferentes mecanismos cognitivos que contribuyen a la ejecución de una tarea compleja. En pocas palabras, si una lesión en la región o en las regiones $\mathrm{A}$ y $\mathrm{B}$ produce déficits en una tarea $\mathrm{A}^{\prime}$, entonces el área o áreas A y B deben ser activadas cuando un individuo sano realiza la tarea $\mathrm{A}^{\prime}$, ya que una lesión en la región o regiones $\mathrm{A}$ y $\mathrm{B}$ produce déficits en la tarea $\mathrm{A}^{\prime}$, pero no en la tarea $\mathrm{B}^{\prime}$, luego el área A o A y B deben ser las áreas activadas cuando una persona sana desempeñe la tarea $\mathrm{A}^{\prime}$, pero no cuando desempeñe la tarea $\mathrm{B}^{\prime}$. Esto se observó en el estudio de Haaland et al. ${ }^{37}$ cuando investigaron secuencias de movimiento en sujetos sanos. Por medio de la resonancia magnética funcional (RMf), se verificó que las regiones frontal izquierda y parietal se activan cuando los sujetos sanos realizan la secuencia de movimientos complejos, pero no con los movimientos simples. Por otra parte, se observó que se organizan secuencias complejas produciendo una gran activación en la región parietal inferior, mientras que la identificación o selección del mecanismo efector apropiado para la secuencia produce una activación en la región parietal superior. ${ }^{32}$

Por lo tanto, los estudios muestran que las asimetrías hemisféricas y un equilibrio óptimo entre los hemisferios podrían ser de utilidad para entender las patologías y enfermedades como el autismo y la esquizofrenia, en las que pueden observarse en parte modulaciones como una especialización hemisférica atípica o incompatible a la integración entre los sistemas neurales disfuncionales, por ejemplo, la disfunción de las neuronas en el circuito espejo. ${ }^{96}$ La regulación de informaciones inter-hemisféricas posiblemente se ejecuta y funciona en varios niveles. El primer nivel (corto plazo) está influenciado, por ejemplo, por factores tales como la atención y el contexto. El segundo nivel (medio plazo) se ve afectado por factores tales como el aprendizaje y la recuperación funcional. Por último, el tercer nivel (largo plazo) se forma por el desarrollo, el envejecimiento, el alto nivel de habilidades y por enfermedades crónicas. Por lo tanto, este punto de vista se opone al punto de vista tradicional en que la lateralización de las funciones motoras es considerada como un proceso estático.

\section{CONCLUSIÓN}

Los hallazgos descritos en la bibliografía muestran un gran interés en estudiar el fenómeno de la regulación de la conducta motora, en particular respecto a la lateralización de las funciones motoras. ${ }^{97}$ Tradicionalmente, los investigadores han estado investigando el predominio del hemisferio izquierdo en el lenguaje y el control del movimiento, mientras que ha sido investigado un dominio del hemisferio derecho de las representaciones espaciales y de la atención. Aunque las áreas especializadas son probablemente predeterminadas, por medio de una combinación de interacciones que una conducta es alcanzada de forma coherente. ${ }^{98}$ En este sentido, un fuerte patrón de conexiones en paralelo a una mayor dependencia de las representaciones del hemisferio izquierdo proporciona la base de un repertorio motor más refinado, y la especialización del hemisferio derecho también parece contribuir a una conducta calificada.

Por otra parte, es por medio de una interacción activa del procesamiento de información, especialmente a través del cuerpo calloso, que ocurre la transferencia de información en procesos como la integración sensitivomotora, la toma de decisiones y la preparación motora. ${ }^{99}$ Como se observa en los temas anteriores, este proceso no se produce de una manera fija, sino en una dinámica impulsada por diversas características de la tarea y el ejecutor, formando de esta manera la conducta motora. Por lo tanto, el patrón de asimetría hemisférica que es la base de la organización de la actividad motora es multifacético y más complejo que una simple dicotomía de funciones. Así, a través de los estudios de neuroimagen, se observaron varios datos en pacientes con lesiones cerebrales asociando los déficits observados en el desempeño de tareas con lesiones 
cerebrales (es decir el hemisferio), lo que demuestra que no se puede interrumpir de forma selectiva las funciones cognitivomotoras especificas, produciendo importantes discusiones de cómo el cerebro representa y regula la conducta motora.

Dentro de este contexto, un punto importante a explorar es cómo contribuye cada hemisferio a las características de la tarea y el ejecutor, lo que permite la oportunidad de observar y comprender mejor la naturaleza del proceso interhemisférico. Estas opiniones no sólo son cruciales para las teorías de control de movimiento, sino también para intervenciones de rehabilitación avanzada en un intento de restaurar la conducta motora de pacientes que sufren de lesiones en el SNC.

\section{REFERENCIAS}

1. Haaland KY. Left hemisphere dominance for movement. Clin Neuropsychol 2006;20:609-622.

2. Matthews PM, Adcock J, Chen Y, Fu S et al. Towards understanding language organization in the brain using fMRI. Hum Brain Mapp 2003;18:239-247.

3. Serrien DJ, Brown P. The functional role of interhemispheric synchronization in the control of bimanual tasks. Exp Brain Res 2002;147:268-272.

4. Serrien DJ, Cassidy MJ, Brown P. The importence of the dominant hemisphere in the organization of bimanual movements. Hum Brain Mapp 2003;18:296-305.

5. Serrien DJ, Fisher RJ, Brown P. Transient increases of synchronized neural activity during movement preparation: influence of cognitive constraints. Exp Brain Res 2003;153:27-34.

6. Serrien DJ, Ivry RB, Swinnen SP. The missing link between action and cognition. Prog Neurobiol 2007;82:95-107.

7. Friston KJ. Models of brain function in neuroimaging. Ann Rev Psychol 2005;56:57-87.

8. Joanette Y, Ansaldo AI, Kahlaoui K, Côté $\mathrm{H}$ et al. Impacto de las lesiones del hemisferio derecho sobre las habilidades lingüísticas: perspectivas teórica y clínica. Rev Neurol 2008;46:481-488.

9. Goble DJ, Brown SH. The biological and behavioral basis of upper limb asymmetries in sensorimotor performance. Neurosci Biobehav Rev 2008;32:598-610.

10. Voets NL, Adcock JE, Flitney DE, Behrens TE et al. Distinct right frontal lobe activation in language processing following left hemisphere injury. Brain 2006;129:754-766.

11. Isaacs KL, Barr WB, Nelson PK, Devinsky O. Degree of handedness and cerebral dominance. Neurology 2006;66:1855-1858.

12. Sveller C, Briellmann RS, Saling MM, Lillywhite L et al. Relationship between language lateralization and handedness in left-hemispheric partial epilepsy. Neurology 2006;67:1813-1817.

13. Homae F, Watanabe $H$, Nakano $T$, Taga G. Prosodic processing in the developing brain. Neurosci Res 2007;59:29-39.

14. Pannekamp A, Weber C, Friederici AD. Prosodic processing at the sentence level in infants. Neuroreport 2006;17:675-178.

15. Vernooij MW, Smits M, Wielopolski PA, Houston GC et al. Fiber density asymmetry of the arcuate fasciculus in relation to functional hemispheric language lateralization in both right- and left-handed healthy subjects: a combined fMRI and DTI study. Neuroimage 2007;35:1064-1076.

16. Pascual A, Huang KL, Neveu J, Préat T. Neuroanatomy: brain asymmetry and long-term memory. Nature 2004;427:605-606.

17. Hatta T. Handedness and the brain: a review of brain-imaging techniques. Magn Reson Med Sci 2007;6:99-112.

18. Skipper JI, Goldin-Meadow S, Nusbaum HC, Small SL. Speech-associated gestures, Broca's area, and the human mirror system. Brain Lang 2007;101:260-277.

19. Lausberg H, Zaidel E, Cruz RF, Ptito A. Speech-independent production of communicative gestures: evidence from patients with complete callosal disconnection. Neuropsychologia 2007;45:3092-3104.

20. Roy AC, Craighero L, Fabbri-Destro M, Fadiga L. Phonological and lexical motor facilitation during speech listening: a transcranial magnetic stimulation study. J Physiol Paris 2008;102:101-105.

21. Watkins K, Paus T. Modulation of motor excitability during speech perception: the role of Broca's area. J Cogn Neurosci 2004;16:978-987.

22. Hauk $O$, Jonhsrude I, Pulvermüller F. Somatotopic representation of action words in the motor and premotor cortex. Neuron 2004;41:301-307.

23. Pulvermüller F, Hauk O, Nilkulin VV, Limoniemi RJ. Functional links between motor and language systems. Eur J Neurosci 2005;21:793-797.

24. Fabbri-Destro M, Rizzolatti G. Mirror neurons and mirror systems in monkeys and humans. Physiology (Bethesda) 2008;23:171-179.

25. Rizzolatti G, Fabbri-Destro M, Cattaneo L. Mirror neurons and their clinical relevance. Nat Clin Pract Neurol 2009;5:24-34.

26. Binkofski F, Buccino G. Motor functions of the Broca's region. Brain Lang 2004;89:362-369.

27. Dominey PF, Hoen M, Blanc, JM, Lelekov-Boissard T. Neurological basis of language and sequential cognition: evidence from simulation, aphasia, and ERP studies. Brain Lang 2003;86:207-225.

28. Dominey PF, Inui T, Hoen M. Neural network processing of natural language: II. Towards a unified model of corticostriatal function in learning sentence comprehension and non-linguistic sequencing. Brain Lang 2008;109:80-92.

29. McGeoch PD, Ramachandran VS. Apraxia, metaphor and mirror neurons. Med Hypotheses 2007;69:1165-1168.

30. Harrington DL, Haaland KY. Hemispheric specialization for motor sequencing: Abnormalities in levels of programming. Neuropsychologia 1991;29:147-163.

31. Kimura D. Left-hemisphere control of oral and brachial movements and their relation to communication. Philos Trans R Soc Lond Biol Sci 1982;298:135-149.

32. Harrington DL, Rao SC, Haaland KY, Bobholz JA et al. Specialized neural systems underlying representation of sequential movements. J Cogn Neurosci 2000;12:56-77.

33. Amunts K, Schleicher A, Zilles, K. Cytoarchitecture of the cerebral cortex-more than localization. Neuroimage 2007;37:1061-1065.

34. Hammond G. Correlates of human handedness in primary motor cortex: a review and hypothesis. Neurosci Biobehav Rev 2002;26:285-92.

35. Gross RG, Grossman M. Update on apraxia. Curr Neurol Neurosci Rep 2008;8:490-496.

36. Haaland KY, Elsinger CL, Mayer AR, Durgerian S et al. Motor sequence complexity and performing hand produce differential patterns of hemispheric lateralization. J Cogn Neurosci 2004;16:621-636.

37. Haaland KY, Prestopnick JL, Knigth RT, Lee RR. Hemispheric asymmetries for kinematic and positional aspects of reaching. Brain 2004;127:1145-1158.

38. Schaefer SY, Haaland KY, Sainburg RL. Ipsilesional motor deficits following stroke reflect hemispheric specializations for movement control. Brain 2007;130:2146-2158.

39. Rinehart JK, Singleton RD, Adair JC, Sadek JR et al. Arm use after left or right hemiparesis is influenced by hand preference. Stroke 2009;40:545-550.

40. Vines BW, Nair D, Schlaug G. Modulating activity in the motor cortex affects performance for the two hands differently depending upon which hemisphere is stimulated. Eur J Neurosci 2008;28:1667-1673.

41. Guye M, Bartolomei F, Ranjeva JP. Imaging structural and functional connectivity: towards a unified definition of human brain organization? Curr Opin Neurol 2008;21:393-403.

42. Müller-Dahlhaus JF, Orekhov Y, Liu Y, Ziemann U. Interindividual variability and age-dependency of motor cortical plasticity induced by paired associative stimulation. Exp Brain Res 2008;187:467-475.

43. Rushworth MF, Taylor PC. TMS in the parietal cortex: updating representations for attention and action. Neuropsychologia 2006;44:2700-2716.

44. Carboni-Roman A, Del Rio Grande D, Capilla A, Maestu F et al. Bases neurobiológicas de las dificultades de aprendizaje. Rev Neurol 2006;42:S171-S175.

45. Jax SA, Coslett HB. Disorders of the perceptual-motor system. Adv Exp Med Biol 2009;629:377-391.

46. O'Shea J, Sebastian C, Boorman ED, Johansen-Berg $\mathrm{H}$ et al. Functional specificity of human premotor-motor cortical interactions during action selection. Eur J Neurosci 2007;26:2085-2095.

47. Króliczak G, Frey SH. A Common network in the left cerebral hemisphere represents planning of tool use pantomimes and familiar intransitive gestures at the hand-independent level. Cereb Cortex 2009;19:2396-2410.

48. Aramaki Y, Honda M, Sadato N. Suppression of the non-dominant motor cortex during bimanual symmetric finger movement: a functional magnetic resonance imaging study. Neuroscience 2006;141:2147-2153. 
49. Cincotta M, Ziemann U. Neurophysiology of unimanual motor control and mirror movements. Clin Neurophysiol 2008;119:744-762.

50. Seitz RJ, Franz M, Azari NP. Value judgments and self-control of action: The role of the medial frontal cortex. Brain Res Rev 2009;60:368-378.

51. Wu X, Chen K, Liu Y, Long Z et al. Ipsilateral brain deactivation specific to the nondominant hand during simple finger movements. Neuroreport 2008;19:483-486.

52. Hickok G, Bellugi U, Klima ES. The neurobiology of sign language and its implications for the neural basis of language. Nature 2002;381:699-702.

53. Hickok G, Poeppel D. The cortical organization of speech processing. Nat Rev Neurosci 2007;8:393-402.

54. Martin JH, Friel KM, Salimi I, Chakrabarty S. Activity- and use-dependent plasticity of the developing corticospinal system. Neurosci Biobehav Rev 2007;31:1125-1135.

55. Mars RB, Piekema C, Coles MG, Hulstijn W et al. On the programming and reprogramming of actions. Cereb Cortex 2007;17:2972-2979.

56. Machado S, Cunha M, Portella CE, Silva JG et al. Integration of cortical areas during performance of a catching ball task. Neurosci Lett 2008;446:7-10.

57. Chase C, Seidler R. Degree of handedness affects intermanual transfer of skill learning. Exp Brain Res 2008;190:317-328.

58. Rossini PM, Dal Forno G. Integrated technology for evaluation of brain function and neural plasticity. Phys Med Rehab Clin North Am 2004;15:263-306.

59. Shabbott BA, Sainburg RL. Differentiating between two models of motor lateralization. J Neurophysiol 2008;100:565-575.

60. Corballis PM. Visuospatial processing and the right-hemisphere interpreter. Brain Cogn 2003;53:171-176.

61. Mesulam MM. Spatial attention and neglect: parietal, frontal and cingulated contributions to the mental representation and attentional targeting of salient extrapersonal events. Philos Trans R Soc Lond Biol Sci 1999;354:1325-1346.

62. Macaluso E, Cherubini A, Sabatini U. Bimanual passive movement: functional activation and inter-regional coupling. Front Integr Neurosci 2007;1:5.

63. Machado S, Cunha M, Portella CE, Silva JG et al. Participación de la corteza parietooccipital en el proceso de integración sensoriomotora: estudio electroencefalográfico. Rev Neurol 2008;47:146-149.

64. Verstynen TD, Dieedrichsen J, Albert N, Aparicio P et al. Ipsilateral motor cortex activity during unimanual hand movements relates to task complexity. J Neurophysiol 2005;93:1209-1222.

65. Sainburg RL. Evidence for a dynamic-dominance hypotesis of handedness. Exp Brain Res 2002;142:241-258.

66. Sun FT, Miller LM, D'esposito M. Measuring temporal dynamics of functional networks using phases spectrum of MRI data. Neuroimage 2005;28:227-237.

67. Halsband U, Lange RK. Motor learning in man: a review of functional and clinical studies. J Physiol Paris 2006;99:414-424.

68. Goldberg E, Podell K, Loveli M. Lateralization of frontal lobe functions and cognitive novelty. J Neuropsychiatry Clin Neurosci 1994;6:371-378.

69. Himmelbach M, Karnath HQ. Goal-directed hand movements are not affected by the biasesd space representation in spatial neglect. J Cogn Neurosci 2003;15:972-980.

70. Debare F, Wenderoth N, Sunaert S, van Hecke P et al. Changes in brain activation during the acquisition of a new bimanual coordination task. Neuropsychologia 2004;42:855-867.

71. Rossini PM, Altamura C, Ferreri F, Melgari JM et al. Neuroimaging experimental studies on brain plasticity in recovery from stroke. Eura Medicophys 2007;43:241-254.

72. Esparza DY, Archambault PS, Winstein CJ, Levin MF. Hemispheric specialization in the co-ordination of arm and trunk movements during pointing in patients with unilateral brain damage. Exp Brain Res 2003;148:488-497.

73. Johansen-Berg H, Matthews PM. Attention to movement modulates activity in sensorimotor areas, including primary motor cortex. Exp Brain Res 2007;142:13-24.

74. Abbruzzese G, Berardelli A. Sensorimotor integration in movement disorders. Mov Disorders 2003;18:231-240.

75. Zeuner KE, Molloy FM. Abnormal reorganization in focal hand dystonia-sensory and motor training programs to retrain cortical function. NeuroRehabilitation 2008;23:43-53.
76. Werhahn KJ, Mortensen J, Van Boven RW, Zeuner KE et al. Enhanced tactile spatial acuity and cortical processing during acute hand deafferentation. Nat Neurosci 2002;5:936-938.

77. Maki Y, Wong KF, Sugiura M, Ozaki T et al. Asymmetric control mechanisms of bimanual coordination: an application of directed connectivity analysis to kinematic and functional MRI data. Neuroimage 2008:42:1295-1304.

78. Duque J, Mazzocchio R, Dambrosia J, Murase N et al. Kinematically specific interhemispheric inhibition operanting in the process of generation of a voluntary movement. Cereb Cortex 2005;15:588-593.

79. De Gennaro L, Cristiani R, Bertini M, Curcio G et al. Handedness is mainly associated with an asymmetry of corticospinal excitability and not of transcallosal inhibition. Clin Neurophysiol 2004;115:1305-1312.

80. Garvey MA, Mall V. Transcranial magnetic stimulation in children. Clin Neurophysiol 2008;119:973-984.

81. Derakhshan I. Laterality of motor control revisited: directionality of callosal traffic and its rehabilitative implications. Top Stroke Rehabil 2005;12:76-82

82. Cardoso de Oliveira S, Gribova A, Donchin O, Bergman H et al. Neural interactions between motor cortical hemispheres during bimanual and unimanual arm movements. Eur J Neurosci 2001;14:1881-1896.

83. Obhi SS, Goodale MA. Bimanual interference in rapid discrete movements is task specific and occurs at multiple levels of processing. J Neurophysiol 2005;94:1861-1868.

84. Maslovat D, Carlsen AN, Ishimoto R, Chua R et al. Response preparation changes following practice of an asymmetrical bimanual movement. Exp Brain Res 2008;190:239-249.

85. Sternad D, Wei K, Diedrichsen J, Ivry RB. Intermanual interactions during initiation and production of rhythmic and discrete movements in individuals lacking a corpus callosum. Exp Brain Res 2007;176:559-574.

86. Varela F, Lachaux JP, Rodríguez E, Martinerie J. The brainweb: phase synchronization and large-scale integration. Nat Rev Neurosci 2001;2:229-239.

87. Sporns O, Chialvo DR, Kaiser M, Hilgetag CC. Organization, development and function of complex brain networks. Trends Cogn Sci 2004;8:418-425.

88. Ioannides AA. Dynamic functional connectivity. Curr Opin Neurobiol 2007;17:161-170.

89. Brovelli A, Ding M, Ledberg A, Chen Y et al. Beta oscillations in a large-scale sensorimotor cortical network: directional influences revealed by Granger causality. Proc Nat Acad Sci USA 2004;101:9849-9854.

90. Zhang Y, Chen Y, Bressler SL, Ding M. Response preparation and inhibition: the role of the cortical sensorimotor beta rhythm. Neuroscience 2008;156:238-246.

91. Fries P. A mechanism for cognitive dynamics: neuronal communication through neuronal coherence. Trends Cogn Sci 2005;9:474-480.

92. Kranczioch C, Athanassiou S, Shen S, Gao G et al. Short-term learning of a visually guided power-grip task is associated with dynamic changes in EEG oscillatory activity. Clin Neurophysiol 2008;119:1419-1430.

93. Hummel F, Gerloff C. Larger interregional synchrony is associated with greater behavioral success in a complex sensory integration task in humans. Cereb Cortex 2005;15:670-678.

94. Andres FG, Mima T, Schulman AE, Dichgans J et al. Functiona coupling of human cortical sensorimotor areas during bimanual skill acquisition. Brain 1999;122:855-870.

95. Sarter M, Berntson GG, Cacioppo JT. Brain imaging and cognitive neuroscience. Toward strong inference in attributing function to structure. Am Psychol 1996;51:13-21.

96. Iacoboni $M$, Dapretto $M$. The mirror neuron system and the consequences of its dysfunction. Nat Rev Neurosci 2006;7:942-951.

97. Bloom JS, Hynd GW. The role of the corpus callosum in interhemispheric transfer of information: excitation or inhibition? Neuropsychol Rev 2005;15:59-71.

98. Gisiger T, Kerszberg M. From a representation of behavior to the concept of cognitive syntax: a theoretical framework. Prog Brain Res 2007;165:463-474.

99. Iacoboni $M$, Zaidel E. Interhemispheric visuo-motor integration in humans: the role of the superior parietal cortex. Neuropsychologia 2004;42:419-425.

Artículo sin conflicto de intereses 